\title{
Incisal Edge
}

National Cancer Institute

\section{Source}

National Cancer Institute. Incisal Edge. NCI Thesaurus. Code C94587.

The cutting edge of an incisor tooth. 